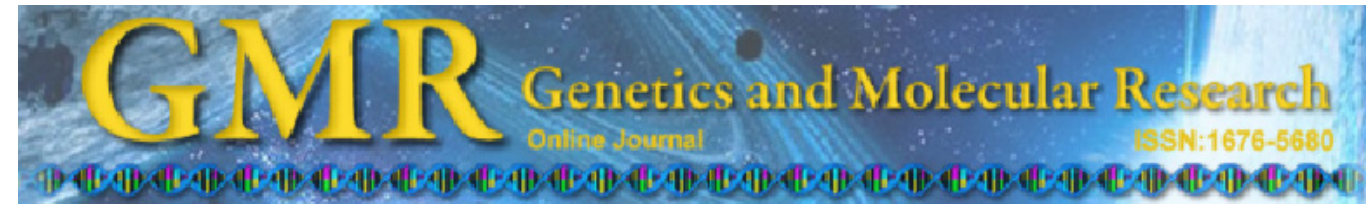

\title{
Assessing molecular and morpho-agronomical diversity and identification of ISSR markers associated with fruit traits in quince (Cydonia oblonga)
}

I. Ganopoulos ${ }^{1,2 *}$, G. Merkouropoulos ${ }^{1 *}$, S. Pantazis $^{3}$, C. Tsipouridis ${ }^{3}$ and A. Tsaftaris ${ }^{1,2}$

${ }^{1}$ Center for Research and Technology Hellas, Institute of Agrobiotechnology, Thermi, Thessaloniki, Greece

${ }^{2}$ Department of Genetics and Plant Breeding, Aristotle University of Thessaloniki, Thessaloniki, Greece ${ }^{3}$ Department of the National Agriculture Research Foundation, Pomology Institute, Naousa, Greece

*These authors contributed equally to this study. Corresponding author: A. Tsaftaris

E-mail: tsaft@certh.gr

Genet. Mol. Res. 10 (4): 2729-2746 (2011)

Received January 19, 2011

Accepted July 25, 2011

Published November 4, 2011

DOI http://dx.doi.org/10.4238/2011.November.4.7

ABSTRACT. Quince is a deciduous tree known to the countries around
the Mediterranean since antiquity. Nowadays, quince is used as an
ornamental plant, and as a rootstock for pear trees, with its fruit being
appreciated mainly for production of jam and sweets rather than for
raw consumption. Quince leaves contain compounds with antioxidant,
antimicrobial and anticancerous properties that have been the focus of
recent research on pharmaceutical and medical uses as well as for food
preservatives. An orchard has been established in Greece, composed
of quince varieties (Cydonia oblonga, $\mathrm{N}=49$ ) collected from different
sites of the country (mainly from home gardens), constituting a
unique quince gene bank collection for southeast Europe. We made a 
phenotypic analysis using 26 morphological plus seven agronomical descriptors coupled with molecular techniques in order to examine the genetic diversity within the collection. Principal component analysis using the 33 descriptors identified 10 components explaining the existence of more than $70 \%$ of the total variation. Subsequent cluster analysis classified most of the previously identified productive varieties of the quince orchard in the same clade of a dendrogram. Molecular analysis generated by 13 inter-simple sequence repeat primers amplified 139 bands, including 109 polymorphic bands, indicating a level of polymorphism of $79 \%$; mean gene diversity was calculated to be 0.309 . Using stepwise multiple regression analysis, a number of markers significantly associated with fire blight susceptibility, yield, mean fruit weight, citric acid content, soluble solid content, and fruit drop were identified. Hence, data extracted by multiple regression analysis could be useful in marker-assisted breeding programs, especially when no previous genetic information is available.

Key words: Cydonia oblonga; Morpho-agronomical descriptors; MRA; Quince; ISSR

\section{INTRODUCTION}

Quince (Cydonia oblonga Miller) is a small deciduous tree that grows up to the height of $8 \mathrm{~m}$, and is related to apple (Malus spp) and pear (Pyrus spp) trees. The quince leaves are ovate to oblong, up to $10 \times 7 \mathrm{~cm}$ in size, possessing many trichomes on the abaxial. The quince flowers bear five petals with hues ranging from white to pink. The fruits are golden yellow and strongly perfumed pomes when mature, with hard flesh and gritty cells.

The name of the genus Cydonia comes from the region of Kydonia at the northwestern coast of Crete, Greece, where the tree has been cultivated since the antiquity. It is believed that quinces were the "golden apples" of Hesperides featured in the eleventh labor of Hercules, and also the fruit that Paris, prince of Troy, offered to Aphrodite, the goddess of love, to gain her favor in seducing Helen and provoking the Trojan war. In ancient Greek ritual, quince fruits were wedding offerings symbolizing fertility.

Quince probably originated from the Caucasian area (Westwood, 1978; USDA, 2009) and spread to the Middle East, Greece and around the Mediterranean. Nowadays, the world yield of quince fruits is produced in a handful of countries among which Turkey is the largest producer followed by China, Iran, Argentina, and Morocco (Postman, 2009). Although the raw fruit is not pleasantly eatable due to its hardness, bitterness, and astringency, it is appreciated for its jam "marmalada" (made of equal amounts of quince puree and sugar), as well as an accompaniment to main dishes and for flavoring pies. Quince is also used in agriculture mainly as a pear rootstock that improves fruit productivity and quality and can be easily propagated using either traditional or modern techniques (Schuch et al., 2010).

Quince is the only species in the genus Cydonia, which falls into the Pomoidaea subfamily of the Rosaceae family. A closely related genus, Chaenomeles (Asian flowering quinces), includes the Japanese quince (C. japonica), which is an ornamental shrubby plant 
with red flowers and small fruits (Hummer and Janick, 2009), that is being cultivated in the north European Baltic countries for fruit production (Rumpunen and Kviklys, 2003).

Long known folk pharmaceutical and medical practices involved quince leaves, after decoction or infusion, for their sedative, antipyretic, anti-diarrheic, and antitussive properties and also for the treatment of various skin diseases (Oliveira et al., 2008). Quince fruit has also been recognized as a natural source for health-promoting compounds with antioxidant, antimicrobial, and antiproliferative properties. For example, of 28 fruits analyzed, the quince fruit was found to be in the top six fruits showing the greatest antioxidant effect (Garcia-Alonso et al., 2004), while the phenolic fraction of quince fruit and jam was also found to contribute significantly to antioxidant potential (Silva et al., 2004). In addition, phenolic compounds in quince fruit and leaves exhibiting antiproliferative properties against the human kidney and colon cancer cells have been reported recently (Carvalho et al., 2010), while eight triterpenoids present in quince fruit peels were reported to show significant antiproliferative activity when tested on murine B16-F1 melanoma cells with ursolic acid to be the most active of all $\left(\mathrm{IC}_{50}=10.2 \mu \mathrm{M}\right)$ (Alesiani et al., 2010). Quince polyphenolic extracts could also be used as biopreservatives inhibiting a wide range of food-borne bacteria responsible for gastroenteritis (Fattouch et al., 2008).

Quince genetic diversity is high. Scaramuzzi (1957) identified 135 cultivars in Europe, USA and USSR conceding, however, that classification was difficult due to the amount of synonyms and the high level of polymorphism (see also Rodriguez-Guisado et al., 2009). Estimation of genetic diversity based on phenotypic analysis of morphological traits, even though it has been used in the past for cultivar development, is greatly influenced by the micro-environment (Shehzad et al., 2009); therefore, it does not provide a globally valid estimate. Other methods involving agronomical measurements, pedigree information, biochemical markers, and molecular markers have also been employed to explore genetic diversity (Fufa et al., 2005). The first attempt to genetically identify and classify quince cultivars using molecular marker technology employed the application of simple sequence repeat (SSR) markers on 20 quince varieties (Yamamoto et al., 2004). Although the SSR markers used by Yamamoto et al. (2004) were originally developed for apples and pears, they separated the quince varieties into two clades on a phenogram, where the varieties used as rootstocks were clustered on one clade of the phenogram, whereas the fruit-producing varieties were on the second clade. The successful application on quince of primers initially designed for apple and pear is not surprising since recent map studies in various plant families, such as Solanaceae, Poaceae, Brassicaceae, and Rosaceae suggest that within each family limited chromosomal rearrangements have occurred resulting in conservation of large chromosome fragments (Celton et al., 2009). Recently, Dumanoglu et al. (2009) evaluated quince fruit traits for six clones of the quince cultivar "Kalecik" for a period of two years and used SSRs to genetically assess clonal variation among the six Kalecik clones. Their results revealed clonal variations within the "Kalecik" cultivar. Genetic studies on quince are expected to increase upon the completion of the apple genome sequencing initiative (Velasco, 2009; Velasco et al., 2010) due to the genetic closeness between the apple and the quince.

The use of the inter-simple sequence repeat (ISSR) primers has been introduced in experimental methodology as an efficient, reproducible, easy to perform, and as a relatively low cost technique to study genetic diversity and genetic structure of a population. The method involves polymerase chain reactions (PCRs) where the ISSR primers recognize and anchor on 
either 5'- or 3'-end of di-, tri-, tetra- or pentanucleotide simple repeats resulting in amplification of the region between such opposite oriented repeats (Reddy et al., 2002). ISSRs have been used for the estimation of genetic variation in various plant species, including Citrus spp (Fang and Roose, 1997), Vitis vinifera L. (Moreno et al., 1998), Plantago major (Wolff and Morgan-Richards, 1998), Olea europea L. (Essadki et al., 2006), Castanea sativa (Mattioni et al., 2008), Morus spp (Kar et al., 2008), Corylus avellana L. (Ferreira et al., 2010), and Prunus avium L. (Ganopoulos et al., 2011).

Although a number of studies have been published concerning quince fruit and leaf chemical composition, as also Dumanoglu et al. (2009) have pointed out, quince has attracted rather minimal attention, both in terms of the traditional scientific approaches (detailed description of quince morphological traits) and the use of the recent scientific methodology as it is represented by the application of molecular techniques. Here, the application of the ISSR method was employed to study the genetic diversity among 49 quince varieties grown in a gene bank quince orchard in Greece. Our approach is the first, to our knowledge, to couple with the molecular marker technology with multivariate analysis concerning morphological traits and agronomical measurements to explore and assess the genetic diversity among the varieties of the collection determining their phylogenetic relationship.

\section{MATERIAL AND METHODS}

\section{Plant materials}

Forty-nine quince (Cydonia oblonga Miller, synonyms: C. vulgaris Pers. and Pyrus cydonia, Linn.) varieties were analyzed in this study (Supplementary Table 1). These varieties had been collected from different parts of Greece between 1980 and 1983 (mainly from home gardens), and were all grafted onto clonal quince rootstock EMA (C. oblonga L.), in a quince orchard established at the Pomology Institute, Department of the National Agriculture Research Foundation Naoussa, Greece, in 1983. The orchard is located at $120 \mathrm{~m}$ a.s.l. and about 25 miles from the sea. The experimental design was completely randomized with three replications of two trees each. The last two trees of each row were not included in the experiments and were used as guard trees. The planting distance was $5 \times 3 \mathrm{~m}$ and the trees were trained to the spindle bush system. All trees had similar size (ca. $3 \mathrm{~m}$ in height) and similar tree vigor. Trees were maintained through standard commercial practices.

\section{Recording of morpho-agronomical descriptors}

A total of 33 morpho-agronomical traits (descriptors) were recorded by direct observation of the trees under field conditions in the quince orchard. These observations were registered in "The European minor fruit tree species database" supporting the "Conservation, evaluation, exploitation, and collection of minor fruit tree species" European project (EC Project GENRES 29), and they are available for public use in the http://www.unifi.it/ueresgen29/ ds6.htm.

The 33 descriptors, comprised of 26 morphological traits plus 7 agronomical traits, together with all recordings are shown in Supplementary Table 1 . The descriptors were firstly recorded using a scale of 1-6 (depending on the trait), and this scale was later converted to the 
binary matrix $(1=$ present, $0=$ absent $)$ for statistical data analysis. The mean value of each variety's quantitative trait was calculated using three replications of two trees each.

\section{Principal component analysis (PCA)}

PCA was performed using the XLSTAT version 2010 software. The data were also used to generate eigenvalues, percentage of the variation accumulated by PCA, and the load coefficient values between the original characters and respective PCA. The first two principal components, which accounted for the highest variation, were used to plot the two-dimensional dispersion of the accessions.

\section{Genomic DNA extraction and PCR amplification conditions}

Genomic DNA was isolated using the CTAB method initially described by Doyle and Doyle (1987). DNA yield was determined using a UV Spectrophotometer (JENWAY 6405 UV/VIS, UK) at A260 nm, whereas DNA purity was estimated according to the A260/A280 ratio. Samples were then diluted to a $30 \mathrm{ng} / \mu \mathrm{L}$ working concentration.

For ISSR analysis amplification was performed in a total volume of $20 \mu \mathrm{L}$ including 30 ng genomic DNA, $200 \mathrm{mM}$ of each dNTPs, 40 pmol primers, $2 \mu \mathrm{L}$ 10X KAPATaq DNA Polymerase buffer, and 1 U KAPATaq DNA Polymerase (KapaBiosystems, Cape Town, South Africa). PCR amplifications were performed in a MasterCycler (Eppendorf, Hamburg, Germany) as follows: an initial step of $5 \mathrm{~min}$ at $94^{\circ} \mathrm{C}$, followed by 35 cycles, each one including $30 \mathrm{~s}$ at $94^{\circ} \mathrm{C}$ for denaturation, $90 \mathrm{~s}$ at $38^{\circ}$ to $55^{\circ} \mathrm{C}$ (depending on the primer) for annealing, and $90 \mathrm{~s}$ at $72^{\circ} \mathrm{C}$ for elongation. A 5-min step at $72^{\circ} \mathrm{C}$ was programmed as a final extension. PCR amplification products were separated by electrophoresis on $1.5 \%$ agarose gel and stained with ethidium bromide. A 100-bp or 1-kb DNA ladder (Invitrogen, USA) was used as a size marker. The selected ISSR primers (Invitrogen; Table 1) were used for PCR amplification. Gels and images were analyzed using the UVIDoc software (UVItec, Cambridge, UK) to quantify signal intensity.

\section{ISSR and principal coordinate (PCOORD) analysis}

Two researchers performed DNA bands scoring, recording all ISSR reproducible fragments irrespective of their intensity. DNA band recordings were typed into a computer file as a binary matrix, where value " 1 " indicated the presence whereas value " 2 " indicated the absence of each fragment. For ISSR analysis, Nei's gene diversity (Nei, 1973) was estimated via the POPGEN 1.32 software (Yeh and Boyle, 1997). The binary data for individuals were subjected to the PCOORD analysis (Peakall and Smouse, 2006), and the first two principal coordinates were plotted to indicate the multilateral genetic relationships among the quince varieties.

\section{Cluster analysis with morpho-agronomical and ISSR data}

The morpho-agronomical and ISSR data were transformed to matrices and were then analyzed by the FreeTree v.0.9.1.50 software (Hampl et al., 2001). Similarity of qualitative 
data was calculated using the Dice similarity index (Nei and Li, 1979) and similarity estimates were analyzed using UPGMA (unweighted pair group method using arithmetic averages). The matrices of mutual coefficients of similarity were calculated using the FreeTree software and then were expressed as dendrograms using the TreeView program (Page, 1996). The robustness of the dendrogram was assessed by bootstrap analysis running 1000 iterations and was also performed by FreeTree. The similarity between matrices based on a different marker system (morpho-agronomical and ISSR data) was calculated using the standardized Mantel coefficient (Mantel, 1967).

\section{RESULTS AND DISCUSSION}

\section{Morpho-agronomical characteristics of the varieties}

Analysis of the recordings shown in Supplementary Table 1 revealed the variability among the varieties. The shape of the lamina is either elliptic (in $51 \%$ of the population studied) or ovate $(36.8 \%)$, with its color being green (57.1\%) or dark green (38.9\%), whereas the size of the lamina is either medium $(53 \%)$ or large $(28.5 \%)$. Fruit, in the majority of the 49 varieties, is either of large $(40.8 \%)$ or medium $(38.7 \%)$ size, having the larger diameter around the middle $(81.6 \%)$, whereas its taste at commercial harvest is regarded as "good" or "very good". Two thirds of the varieties flower (time of flowering) late, while the remaining varieties exhibit early or medium flowering. Forty-three of the 49 varieties, fully flower (descriptor: date of fully flower) within four consecutive days, leaving five varieties with an earlier flowering time and one variety with a late flowering time. The tree vigor was estimated either as strong or medium in 42 of the 49 varieties (85.7\%). Yield and fruit drop were found to be inversely proportional descriptors with high variability; yied ranges from 1 $\mathrm{kg} /$ tree (varieties 11128 and 11129) to $115 \mathrm{~kg} /$ tree (variety 11111), whereas fruit drop ranges between $1.3 \%$ (variety 11113 ) to $65.1 \%$ (variety 11105 ). One of the morphological descriptors (fruit color) was similar for all varieties (greenish-yellow); therefore, it was not used in the construction of the relevant dedrograms since it did not confer to the essential variability.

In a previous study, we have investigated the postharvest performance of the same 49 quince varieties of the Greek orchard (Thomidis et al., 2004). Focusing on agronomical descriptors such as productivity, fruit drop, fire blight susceptibility, scald, and cool storage only, we had managed to identify varieties with outstanding performance regarding productivity, and improved performance regarding the remaining descriptors. The main goal of the present study was to investigate the genetic diversity among the 49 quince varieties of the Greek orchard based on the analysis of the 26 morphological plus 7 agronomical descriptors (thus, 33 phenotypic descriptors in total), coupled with molecular analysis with the application of the ISSR marker technology. It should be mentioned that most of the measurements concerning the common agronomical descriptors between the previous study (Thomidis et al., 2004) and the study herein were comparable but were not the same, since the recordings were taken in different years. Annual variation in recordings of traits such as fruit weight, length and diameter was reported to occur for quince cultivar Kalecik (Dumanoglu et al., 2009).

\section{Multivariate analysis of the $\mathbf{3 3}$ descriptors}

PCA is a descriptive method that reduces a large number of variables that are used to 
characterize individual accessions, into a small number of units or components (Atchley and Bryant, 1975). Although the variables within each component are not correlated, the resulting components are highly correlated and are used to explain different parts of the total variance among the accessions. PCA analysis has been performed in the past in many plant species including trees.

PCA analysis was performed using the total of the 33 descriptors, identifying $10 \mathrm{com}$ ponents to explain more than $70 \%$ of the total variation. The first principal component (PC1) accounted for $14.863 \%$ of the total variation and, according to the Kaiser criterion (retaining only those factors with eigenvalues greater than 1), it was mainly influenced by yield and entity of production. The second component (PC2) explained for $8.971 \%$ of the total variation and it was mainly correlated to descriptors such as spacing between petals and pubescence of the lower side of the leaf blade. It seems therefore that the first component is correlated mainly to quantitative descriptors related to productivity, whereas the second component is largely influenced by morphological descriptors (Figure 1). PC3 accounted for $8.271 \%$ of the variation and was positively correlated to descriptors on fruit characteristics, such as the fruit taste at physiological harvest and the fruit shape. The fourth component (PC4) explained 7.618\% of the total variation and was dominated by citric acid content and color of upper side leaf blade. The fifth component (PC5) was determined by mean fruit weight, position of maximum diameter in fruit and leaf blade shape, and accounted for $6.201 \%$ of the total variation. PC6, 7 , 8,9 , and 10 explained an additional $5.829,5.325,5.075,4.501$, and $3.945 \%$ of the total variation. PC6 was influenced by characters related to the flower, such as shape of petal, position of stigma compared to anthers, color of flower, and the date of full flowering. PC7 was dominated by soluble solid content and dimension of pistil cavity of the fruit. PC8 was correlated mainly to fruit taste at commercial harvest, time of flowering and soluble solid content, while PC9 and PC10 were related to descriptors such as fire blight susceptibility and diameter of crown of the flower, and fruit size, pubescence of lower side of leaf blade, fruit firmness, and time of flowering, respectively. A total of another 22 principal components, which were represented with variations ranging from 3.5 to $0.04 \%$, were considered to be less important to the overall variability (all the correlations between variables and factors are shown in Supplementary Table 2).

\section{Morpho-agronomical phylogenetic analysis}

The standardized morpho-agronomical data, as defined by the 33 descriptors, was used to calculate the Euclidean distances between the 49 quince varieties, producing a dendrogram where the varieties are classified into two major clusters, Cluster A and Cluster B (Figure 2). Cluster A is comprised of 28 varieties whereas Cluster B is comprised of 20 varieties. One variety (11131) was left unclassified. Cluster A is subclassified into three subclusters of sixteen, seven and four varieties (variety 11134 was left unclassified), whereas Cluster B is subdivided into two subclusters of twelve and seven varieties (variety 11126 was left unclassified).

Six of the seven most productive quince varieties (Thomidis et al., 2004) in the gene bank collection are clustered in Cluster B (variety 11111 is not included), with four of these varieties being on the same clade of subcluster B1. With the exception of fruit size and fruit shape these varieties exhibit similar morphological traits, whereas their agronomical traits are also comparable (high values for yield and mean fruit weight, and low values for fire blight susceptibility and fruit drop). 


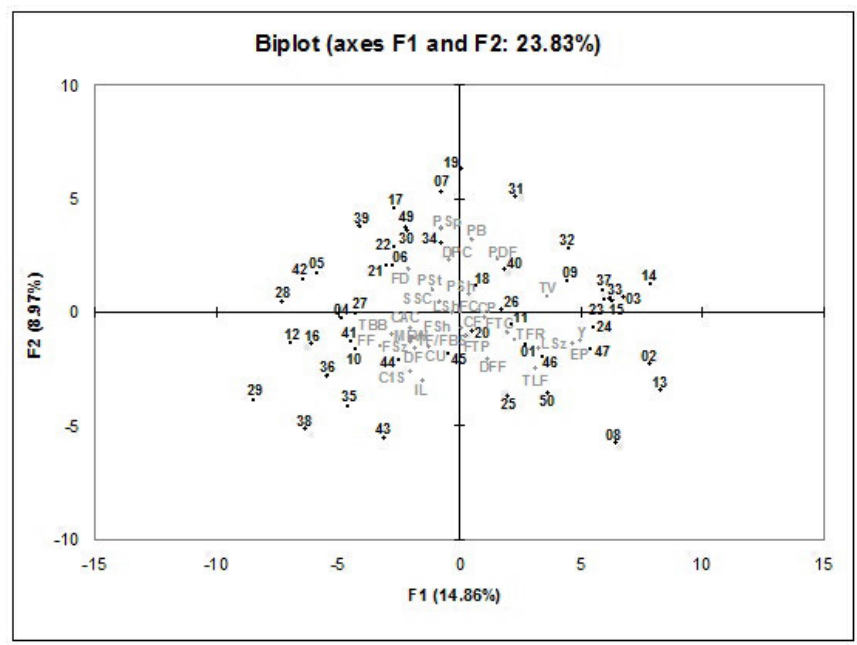

Figure 1. Biplot of the first two principal components showing the distribution of the 49 quince varieties (for convenience, only the last two digits of each variety's code number are shown in black fonts) in accordance with the 33 morpho-agronomical descriptors (in light gray fonts) used. Abbreviations of the descriptors are shown in Supplementary Table 1.

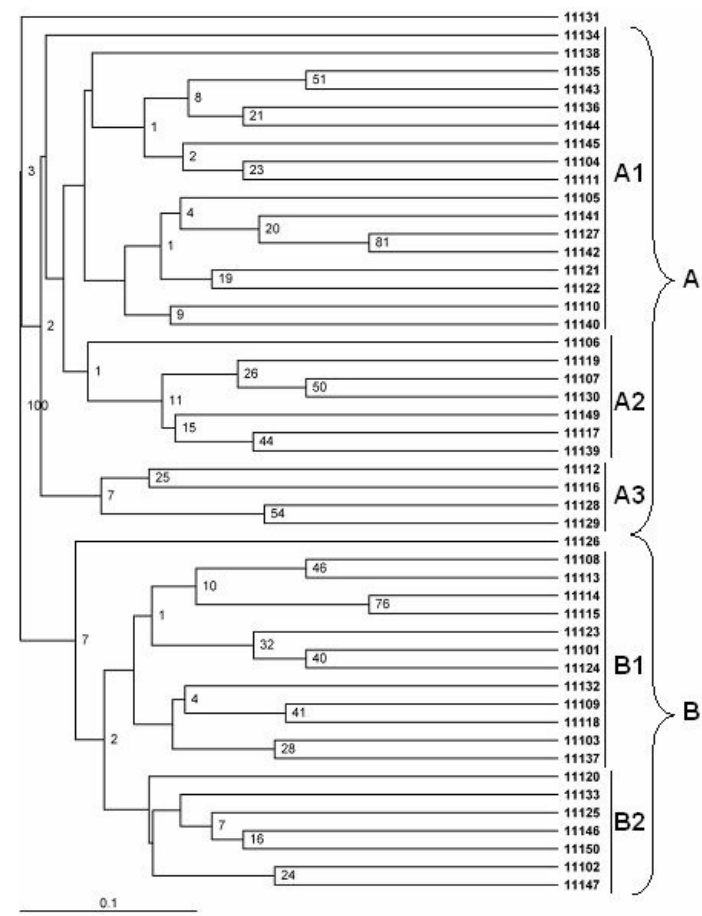

Figure 2. UPGMA dedrogram constructed using the 33 morpho-agronomical descriptors available for the 49 quince varieties of the collection. The scale shown at the bottom of the dendrogram is the genetic distance as calculated according to Nei and $\mathrm{Li}$ (1979). Numbers in the fork positions indicate bootstrap values (percentage of 1000 replicates). 


\section{ISSR analysis}

To our knowledge, prior to the present report, the only published molecular study concerning quince varieties was the Yamamoto study where a number of SSRs developed for apples and pears had been used to investigate the genetic closeness of 20 quince varieties (Yamamoto et al., 2004). Eight of the varieties that were studied by Yamamoto et al. were used for fruit production, ten were used as rootstocks for pear (one of the varieties was used for fruit production and as a rootstock for pear), while three varieties were used for other purposes. The Yamamoto study resulted in the construction of a phenogram with separation of the quince varieties to those used as rootstocks and those used for fruit production. In a recent report, the clonal diversity within quince cultivar Kalecik was analyzed by using seven SSR loci and detecting genetic differences among the clones (Dumanoglu et al., 2009).

In the present study, 18 ISSR primers, chosen from the University of British Columbia collection, have been tested on 49 genomic DNAs extracted from equal number of quince plants from the gene bank quince collection. Thirteen of these primers resulted in the generation of multiple banding profile (Table 1), while five primers produced smear. The number of bands that have been produced per primer ranged from six (primer UBC808) to fourteen (primer UBC814 and primer UBC834), resulting in a total of 139 amplified DNA bands for all varieties. A total of 109 polymorphic bands were detected by the 13 primers both within and between the varieties, raising the level of polymorphism to $79.15 \%$. In the subsequent analysis, the detected polymorphic markers (bands) were used to calculate first the similarity coefficient matrix and then to construct a similarity dendrogram using the UPGMA cluster algorithm. In this similarity dendrogram, the 49 quince varieties were classified into five clusters, with two major clusters of 28 and 12 varieties each (Figure 3, Clusters A and B, respectively), and three smaller clusters comprised of two to three varieties (Figure 3, Clusters C, D and E); one variety was unclassified (11150). Six varieties (11102, 11103, 11108, 11111, 11113, and 11115) of the seven most productive quince varieties in the collection (Thomidis et al., 2004), are classified in Cluster A, leaving one variety (11114) in Cluster D. Judging by their position on the dendrogram, varieties 11103 and 11108 share high level of genetic relationship.

The PCOORD scatter plot (Figure 4) further supported the dendrogram (shown in Figure 3) results in a robust way, considering the high percentage of the total genetic diversity (47.21\%) that was accounted for in low multivariate space. In a comparable study of apple cultivars, $40.43 \%$ could be accounted for by the first two coordinates (Song et al., 2006).

When the Mantel test was applied to the matrix of the Nei's genetic distances and the matrix of morpho-agronomical descriptors, slight and significant correlation was observed between these two matrices, with a $\mathrm{P}<0.01(\mathrm{R}=0.34)$, indicating that the clustering of varieties based on morpho-agronomical descriptors is consistent with the clustering derived from the molecular analysis.

The diversity of the gene bank collection was represented with Nei's gene diversity. Data for gene diversity for all the 49 quince varieties were analyzed using 13 polymorphic ISSR markers and their corresponding mean value was calculated to 0.309 (Table 1). The results provide guidance for future effective use of these molecular methods in the genetic analysis of quince. The collection of primers used in this study gave a reasonable number of amplification products for the genetic diversity analysis. This study reveals the great importance of ensuring the differentiation of quince varieties and their application for certification purposes. 
I. Ganopoulos et al.

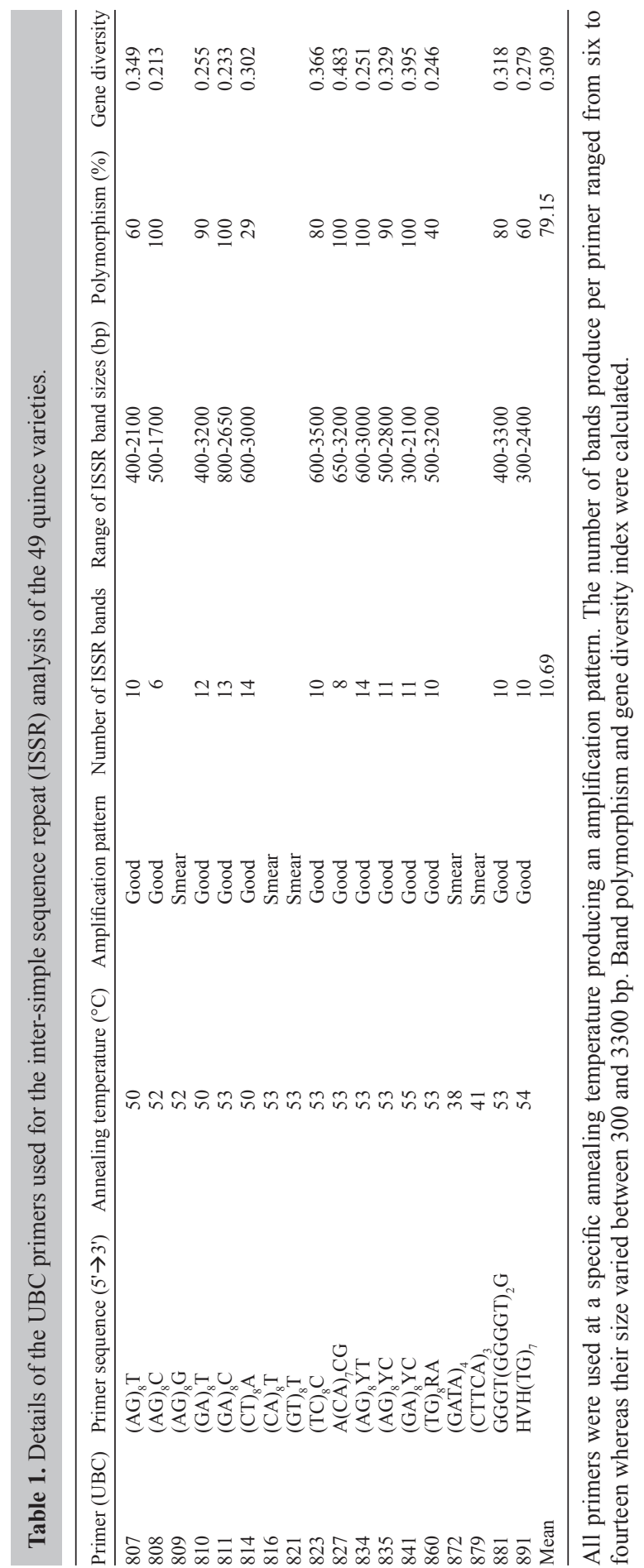




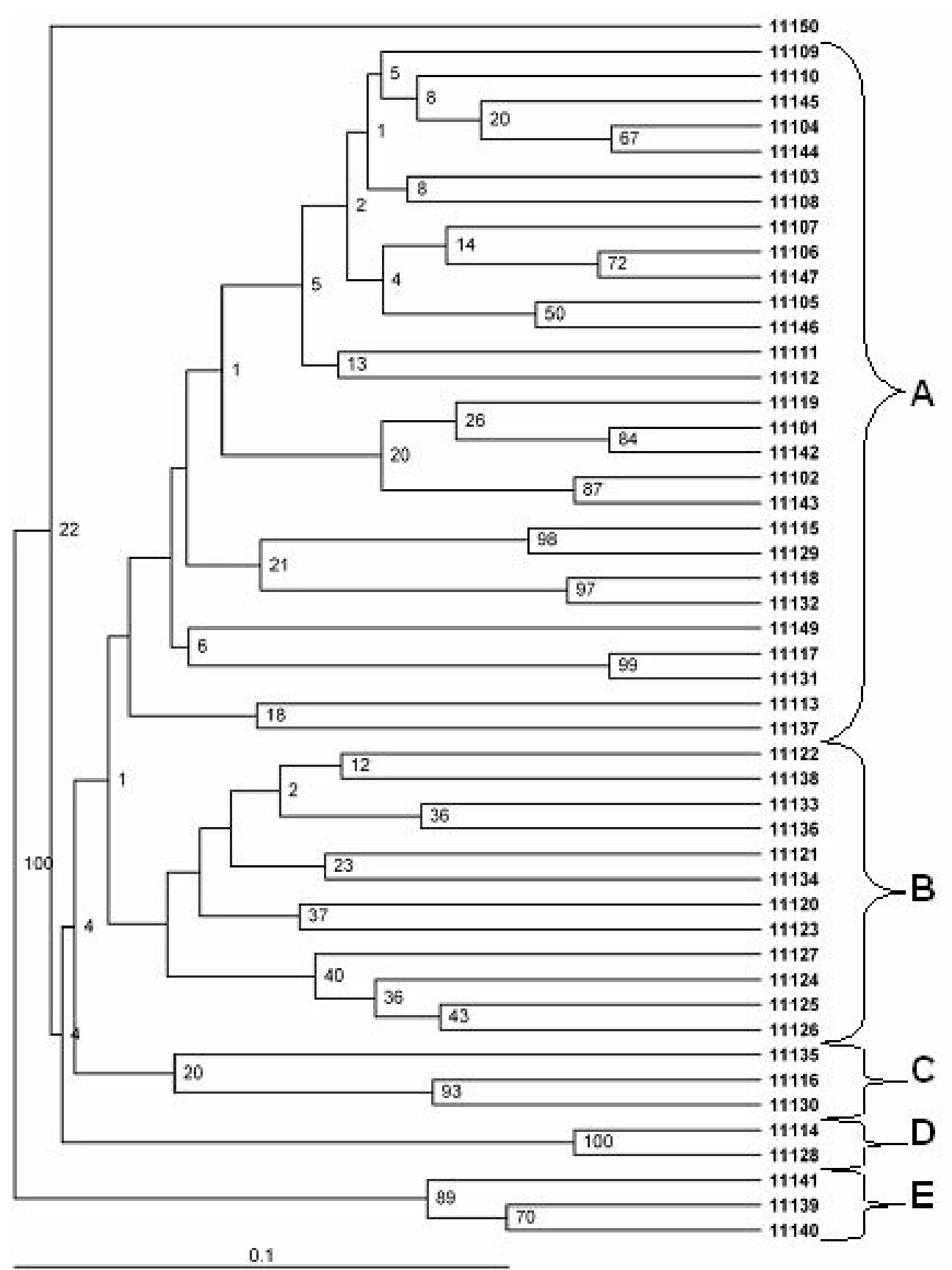

Figure 3. UPGMA dedrogram constructed from ISSR marker analysis showing the genetic relationships among the 49 quince varieties of the quince collection. The scale shown at the bottom of the dendrogram is the genetic distance as calculated according to Nei and Li (1979). Numbers in the fork positions indicate bootstrap values (percentage of 1000 replicates). 


\section{Principal Coordinates}

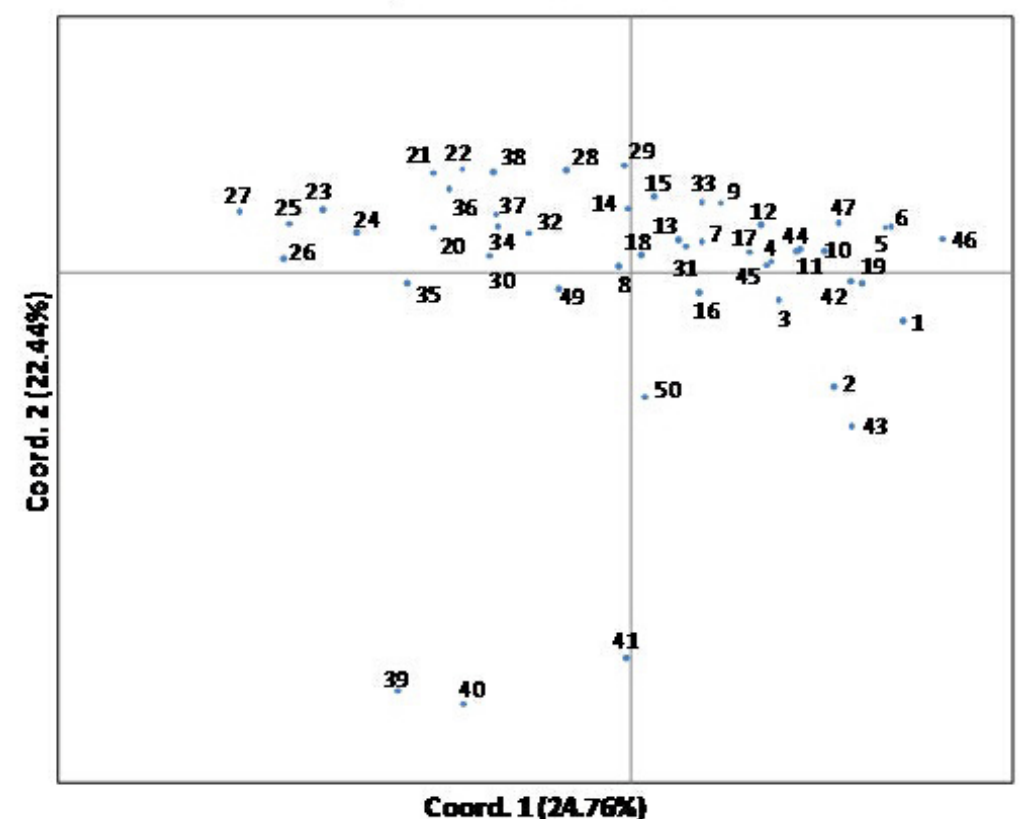

Figure 4. Principal coordinates analysis (PCOORDA) plot based on ISSR data. The two principal coordinates account for an accumulate variation of $47.21 \%$. For convenience, only the last two digits of each variety's code number are shown.

Stepwise multiple regression analysis identified groups of polymorphic markers showing statistically significant correlation to specific agronomical descriptors, with the number of polymorphic markers varying from three to six (Table 2). Three polymorphic markers were identified for fire blight susceptibility, four for yield, five for mean fruit weight, citric acid content, and soluble solid content, and six for fruit drop. One polymorphic band $\left(\mathrm{UBC} 827_{2500}\right)$ was found to be associated with two different descriptors (fire blight susceptibility and fruit drop), whereas all the remaining polymorphic bands were correlated to single descriptors. ISSR markers UBC $811_{2600}, \mathrm{UBC}_{22} 3_{2300}$ and UBC891 500 exhibited high correlation $(\mathrm{P}<0.001)$ to citric acid content, soluble solid content and fire blight susceptibility, respectively, whereas markers $\mathrm{UBC} 80_{3200}$ and $\mathrm{UBC} 81_{2000}$ showed correlation to fruit drop $(\mathrm{P}<0.01)$ and yield $(\mathrm{P}<0.01)$, respectively.

The methods described in this study provide a reliable and easy to perform analysis in order to identify the promising cultivars at the early stages of breeding programs. Previously, an association analysis approach was adopted by Virk et al. (1996) to establish two qualitative traits, and by Kar et al. (2008) to identify a suite of markers linked to biochemical traits in mulberry tree. MRA approach is a convenient tool for tree crops and a quick method for establishing marker-trait association avoiding the need for mapping populations. The markers identified in this study can be used for MAS breeding programs. 


\begin{tabular}{|c|c|c|c|c|c|}
\hline & Markers & Sum of $R^{2}$ & $\mathrm{R}^{2}$ change & F-change & $P$ value of $F$-change \\
\hline \multirow[t]{3}{*}{ FBS } & UBC891 & 0.355 & 0.355 & 17.060 & 0.000 \\
\hline & +UBC811 & 0.469 & 0.114 & 6.445 & 0.017 \\
\hline & $+\mathrm{UBC} 827_{2500}^{200}$ & 0.547 & 0.078 & 4.995 & 0.033 \\
\hline \multirow[t]{4}{*}{ Y } & UBC $841_{2500}$ & 0.204 & 0.204 & 8.952 & 0.005 \\
\hline & $+\mathrm{UBC} 823_{650}$ & 0.331 & 0.128 & 6.496 & 0.015 \\
\hline & +UBC $811_{9000}^{650}$ & 0.435 & 0.104 & 6.059 & 0.019 \\
\hline & $+\mathrm{UBC} 811_{1700}$ & 0.507 & 0.072 & 4.663 & 0.038 \\
\hline \multirow[t]{5}{*}{ MFW } & $\mathrm{UBC} 807_{2000}$ & 0.192 & 0.192 & 8.315 & 0.007 \\
\hline & $+\mathrm{UBC} 810^{200}$ & 0.350 & 0.158 & 8.266 & 0.007 \\
\hline & $+\mathrm{UBC} 808_{1600}^{600}$ & 0.442 & 0.092 & 5.422 & 0.026 \\
\hline & $+\mathrm{UBC} 835_{1500}^{1600}$ & 0.553 & 0.112 & 7.988 & 0.008 \\
\hline & + UBC $841_{1500}^{1500}$ & 0.626 & 0.073 & 6.077 & 0.019 \\
\hline \multirow[t]{5}{*}{$\mathrm{CAC}$} & UBC811 2600 & 0.343 & 0.343 & 18.302 & 0.000 \\
\hline & $+\mathrm{UBC} 827_{2300}^{260}$ & 0.434 & 0.091 & 5.439 & 0.026 \\
\hline & +UBC $881_{1850}^{2300}$ & 0.566 & 0.132 & 10.016 & 0.003 \\
\hline & $+\mathrm{UBC} 835_{1800}$ & 0.640 & 0.074 & 6.551 & 0.015 \\
\hline & $+\mathrm{UBC} 835_{700}^{1800}$ & 0.691 & 0.051 & 5.155 & 0.030 \\
\hline \multirow[t]{5}{*}{$\mathrm{SSC}$} & UBC $891_{750}^{700}$ & 0.165 & 0.165 & 6.895 & 0.013 \\
\hline & +UBC $841_{700}$ & 0.306 & 0.141 & 6.932 & 0.013 \\
\hline & + UBC823 ${ }_{2300}^{700}$ & 0.520 & 0.214 & 14.706 & 0.001 \\
\hline & $+\mathrm{UBC} 841_{2100}^{2300}$ & 0.610 & 0.090 & 7.360 & 0.011 \\
\hline & $+\mathrm{UBC} 881_{1450}^{2100}$ & 0.680 & 0.070 & 6.748 & 0.014 \\
\hline \multirow[t]{6}{*}{ FD } & UBC $860_{3200}^{140}$ & 0.235 & 0.235 & 10.731 & 0.002 \\
\hline & +UBC814 & 0.383 & 0.148 & 8.168 & 0.007 \\
\hline & + UBC $841_{1300}^{9300}$ & 0.480 & 0.098 & 6.193 & 0.018 \\
\hline & $+\mathrm{UBC} 807_{2100}^{1300}$ & 0.557 & 0.077 & 5.570 & 0.025 \\
\hline & $+\mathrm{UBC} 827_{3000}^{2100}$ & 0.616 & 0.059 & 4.767 & 0.037 \\
\hline & +UBC $827_{2500}^{3500}$ & 0.684 & 0.068 & 6.420 & 0.017 \\
\hline
\end{tabular}

$\overline{\mathrm{FBS}}=$ fire blight susceptibility; $\mathrm{Y}=$ yield; $\mathrm{MFW}=$ mean fruit weight $\mathrm{CAC}=$ citric acid content; $\mathrm{SSC}=$ soluble solid content; FD = fruit drop.

\section{CONCLUSION}

Quince is an agronomically important species with different uses and rather limited information concerning its morphology and genetic variability. The importance of the utilization of morpho-agronomical and ISSR markers in the management of the quince gene bank collection was established in this study improving the conservation and management of the relevant genetic resources. The genetic diversity data obtained are helpful in the identification of duplicate varieties, verification of synonyms and homonyms and determination of misidentified varieties. ISSR markers can be used in order to specify the gaps in the gene pools and organize the future additions. The application of the ISSR approach enables us to predict positive correlation between molecular marker data and morpho-agronomical descriptors on a species that is expected to attract much attention in the near future. The completion of the genome sequencing of a closely related species, such as the apple, will facilitate the wide use of molecular study on quince in the forthcoming years.

\section{REFERENCES}

Alesiani D, Canini A, D’Abrosca B, DellaGreca M, et al. (2010). Antioxidant and antiproliferative activities of phytochemicals from Quince (Cydonia vulgaris) peels. Food Chem. 118: 199-207. 
Atchley WR and Bryant EH (1975). Multivariate Statistical Methods. Benchmark Papers in Systematic and Evolutionary Biology 1 Dowden. Hutchinson and Ross, Stroudsberg.

Carvalho M, Silva BM, Silva R, Valentao P, et al. (2010). First report on Cydonia oblonga Miller anticancer potential: differential antiproliferative effect against human kidney and colon cancer cells. J. Agric. Food Chem. 58: 33663370.

Celton JM, Chagne D, Tustin SD, Terakami S, et al. (2009). Update on comparative genome mapping between Malus and Pyrus. BMC Res. Notes 2: 182.

Doyle JJ and Doyle JL (1987). A rapid DNA isolation procedure for small quantities of fresh leaf tissue. Phytochem. Bull. 19: 11-15.

Dumanoglu H, Gunes NT, Aygun A, San B, et al. (2009). Analysis of clonal variations in cultivated quince (Cydonia oblonga 'Kalecik') based on fruit characteristics and SSR markers. New Zeal. J. Crop Hort. 37: 113-120.

Essadki M, Ouazzani N, Lumaret R and Moumni M (2006). ISSR variation in olive-tree cultivars from Morocco and other western countries of the Mediterranean Basin. Genet. Res. Crop Evol. 53: 475-482.

Fang DQ and Roose ML (1997). Identification of closely related citrus cultivars with inter-simple sequence repeat markers. Theor. Appl. Genet. 95: 408-417.

Fattouch S, Sadok S, Raboudi-Fattouch F and Ben Slama M (2008). Damage inhibition during refrigerated storage of mackerel (Scomber scombrus) fillets by a presoaking in quince (Cydonia oblonga) polyphenolic extract. Int. J. Food Sci. Technol. 43: 2056-2064.

Ferreira JJ, Garcia-Gonzales C, Tous J and Rovira M (2010). Genetic diversity revealed by morphological traits and ISSR markers in hazelnut germplasm from northern Spain. Plant Breed. 129: 435-441.

Fufa H, Baenziger PS, Beecher BS, Dweikat I, et al. (2005). Comparison of phenotypic and molecular marker-based classifications of hard red winter wheat cultivars. Euphytica 145: 133-146.

Ganopoulos IV, Kazantzis K, Chatzicharisis I, Karayiannis I, et al. (2011). Genetic diversity, structure and fruit trait associations in Greek sweet cherry cultivars using microsatellite based (SSR/ISSR) and morpho-physiological markers. Euphytica 18: 237-251.

Garcia-Alonso M, Pascual-Teresa S, Santos-Buelga C and Rivas-Gonzalo JC (2004). Evaluation of the antioxidant properties of fruits. Food Chem. 84: 13-18.

Hampl V, Pavlicek A and Flegr J (2001). Construction and bootstrap analysis of DNA fingerprinting-based phylogenetic trees with the freeware program FreeTree: application to trichomonad parasites. Int. J. Syst. Evol. Microbiol. 51: 731-735.

Hummer KE and Janick J (2009). Rosaceae: Taxonomy, Economic Importance, Genomics. In: Genetics and Genomics of Rosaceae (Folta KM and Gardiner SE, eds.). Springer, New York, 1-17.

Kar PK, Srivastava PP, Awasthi AK and Urs SR (2008). Genetic variability and association of ISSR markers with some biochemical traits in mulberry (Morus spp.) genetic resources available in India. Tree Genet. Genom. 4: 75-83.

Mantel N (1967). The detection of disease clustering and a generalized regression approach. Cancer Res. 27: 209-220.

Mattioni C, Cherubini M, Micheli E, Villani F, et al. (2008). Role of domestication in shaping Castanea sativa genetic variation in Europe. Tree Genet. Genom. 4: 563-574.

Moreno S, Martin JP and Ortiz JM (1998). Inter-simple sequence repeats PCR for characterization of closely related grapevine germplasm. Euphytica 101: 117-125.

Nei M (1973). Analysis of gene diversity in subdivided populations. Proc. Natl. Acad. Sci. 70: 3321-3323.

Nei M and Li WH (1979). Mathematical model for studying genetic variation in terms of restriction endonucleases. Proc. Natl. Acad. Sci. 76: 5269-5273.

Oliveira AP, Pereira JA, Andrade PB, Valentao P, et al. (2008). Organic acids composition of Cydonia oblonga Miller leaf. Food Chem. 111: 393-399.

Page RD (1996). TreeView: an application to display phylogenetic trees on personal computers. Comput. Appl. Biosci. 12: 357-358.

Peakall R and Smouse PE (2006). Genalex 6: genetic analysis in Excel. Population genetic software for teaching and research. Mol. Ecol. Notes 6: 288-295.

Postman J (2009). Cydonia oblonga: The Unappreciated Quince. The magazine of the Arnold Arboretum, Arnoldia, 2-9.

Reddy MP, Sarla N and Siddiq EA (2002). Inter simple sequence repeat (ISSR) polymorphism and its application in plant breeding. Euphytica 128: 9-17.

Rodriguez-Guisado I, Hernandez F, Melgarejo P, Legua P, et al. (2009). Chemical, morphological and organoleptical characterisation of five Spanish quince tree clones (Cydonia oblonga Miller). Sci. Hortic. 122: 491-496.

Rumpunen K and Kviklys D (2003). Combining ability and patterns of inheritance for plant and fruit traits in Japanese quince (Chaenomeles japonica). Euphytica 132: 139-149.

Scaramuzzi F (1957). Contributo allo Studio delle cultivar di cotogno da frutto. Riv. Fruttic. Ortofloric. Ital. 41: 575-615.

Genetics and Molecular Research 10 (4): 2729-2746 (2011)

CFUNPEC-RP www.funpecrp.com.br 
Schuch MW, Cellini A, Masia A and Marino G (2010). Aluminium-induced effects on growth, morphogenesis and oxidative stress reactions in in vitro cultures of quince. Sci. Hortic. 125: 151-158.

Shehzad T, Okuizumi H, Kawase M and Okuno K (2009). Development of SSR-based sorghum (Sorghum bicolor (L.) Moench) diversity research set of germplasm and its evaluation by morphological traits. Genet. Res. Crop Evol. 56: 809-827.

Silva BM, Andrade PB, Valentao P, Ferreres F, et al. (2004). Quince (Cydonia oblonga Miller) fruit (pulp, peel, and seed) and jam: antioxidant activity. J. Agric. Food Chem. 52: 4705-4712.

Song Y, Zhai H, Yao Y-X, Li M, et al. (2006). Analysis of genetic diversity of processing apple varieties. Agr. Sci. China 5: 745-750.

Thomidis T, Tsipouridis C, Isaakidis A and Michailides Z (2004). Documentation of field and postharvest performance for a mature collection of quince (Cydonia oblonga) varieties in Imathia, Greece New Zeal. J. Crop Hort. 32: 243-247.

USDA (2009). Germplasm resources information network- (GRIN) Online Database, National Germplasm Resources Laboratory. Available at [http://www.ars-grin.gov/cgi-bin/npgs/html/taxon.pl?12779]. Accessed February 5, 2009.

Velasco R (2009). The Golden Delicious Apple Genome: An International Whole Genome Sequencing Initiative. Plant and Animal Genomes XVII Conference, San Diego, W211.

Velasco R, Zharkikh A, Affourtit J, Dhingra A, et al. (2010). The genome of the domesticated apple (Malus x domestica Borkh.). Nat. Genet. 42: 833-839.

Virk PS, Ford-Lloyd BV, Jackson MT, Pooni HS, et al. (1996). Predicting quantitative variation within rice germplasm using molecular markers. Heredity 76: 296-304.

Westwood MN (1978). Temperate-Zone Pomology. WH Freeman and Company, San Francisco.

Wolff K and Morgan-Richards M (1998). PCR markers distinguish Plantago major subspecies. Theor. Appl. Genet. 96: 282-286.

Yamamoto T, Kimura T, Soejima J, Sanada T, et al. (2004). Identification of quince varieties using SSR markers developed from pear and apple. Breed. Sci. 54: 239-244.

Yeh FC and Boyle TJB (1997). POPGENE, Version 1.1. Department of Renewable Resources, University of Alberta, Edmonton. 


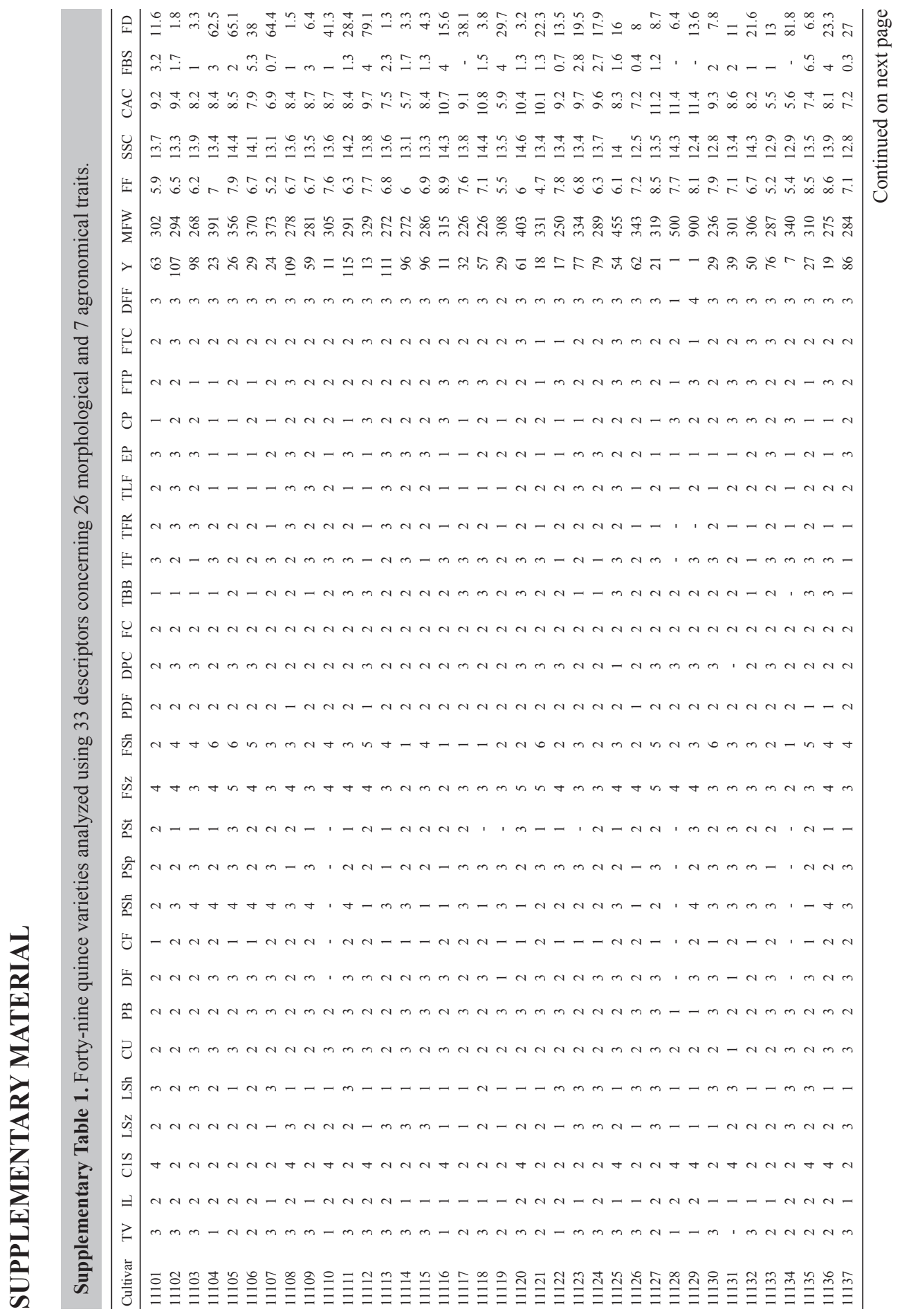




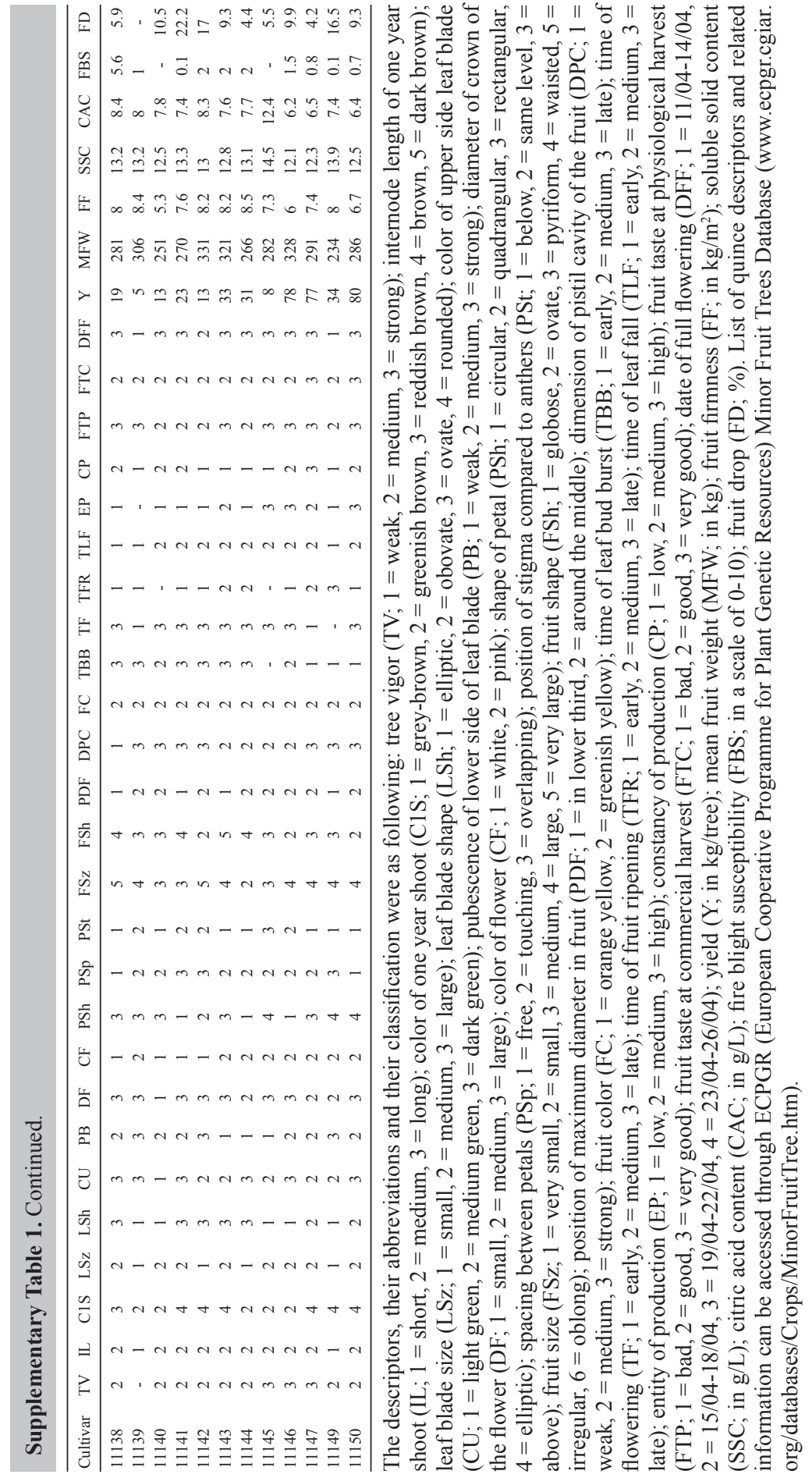




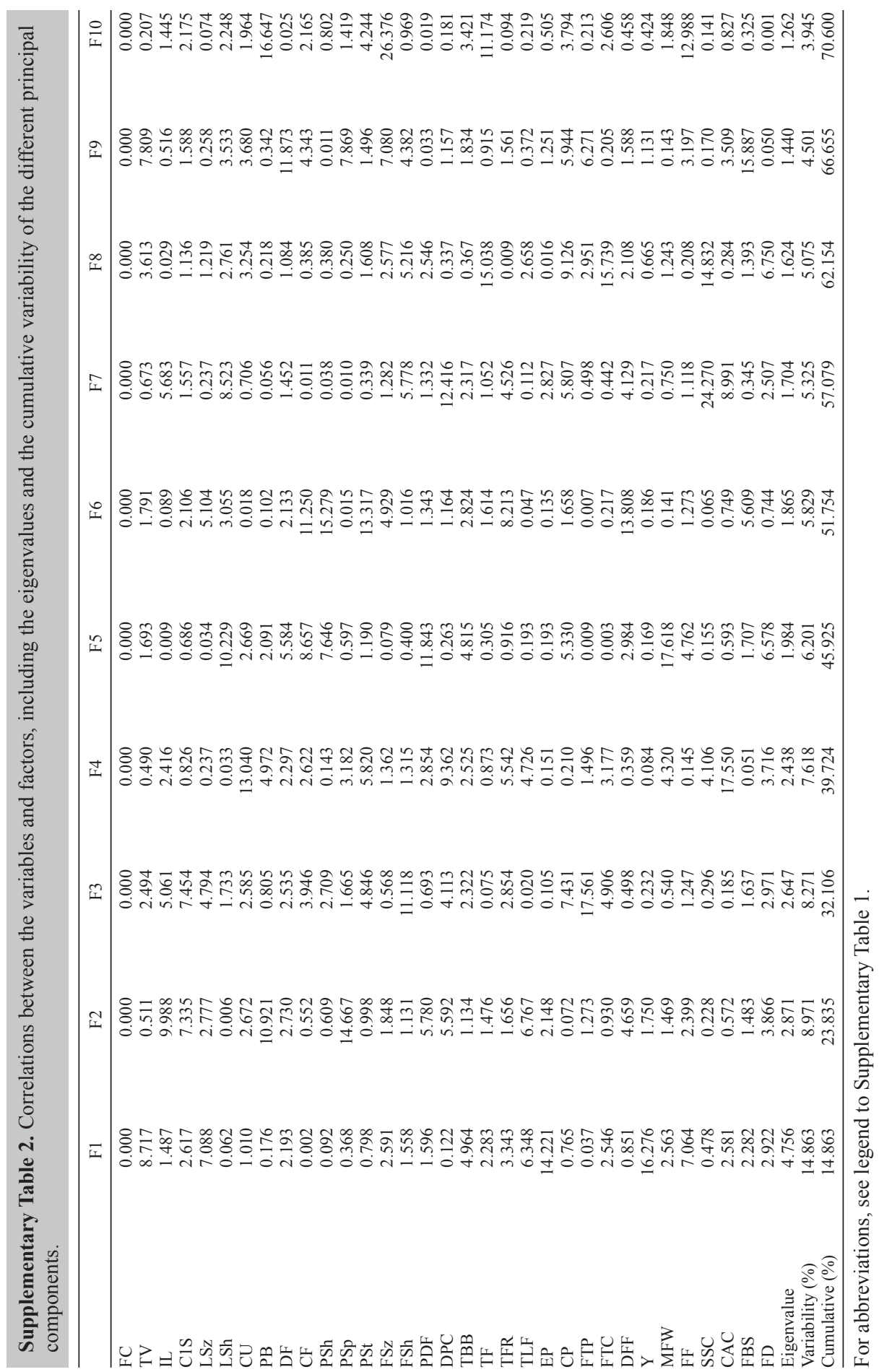

